\title{
Article
}

\section{Activation of proton translocation by respiratory Complex I}

\author{
Nikolai P. Belevich, Christoph von Ballmoos, and Marina Verkhovskaya
}

Biochemistry, Just Accepted Manuscript • DOI: 10.1021/acs.biochem.7b00727 • Publication Date (Web): 29 Sep 2017

Downloaded from http://pubs.acs.org on October 1, 2017

\section{Just Accepted}

"Just Accepted" manuscripts have been peer-reviewed and accepted for publication. They are posted online prior to technical editing, formatting for publication and author proofing. The American Chemical Society provides "Just Accepted" as a free service to the research community to expedite the dissemination of scientific material as soon as possible after acceptance. "Just Accepted" manuscripts appear in full in PDF format accompanied by an HTML abstract. "Just Accepted" manuscripts have been fully peer reviewed, but should not be considered the official version of record. They are accessible to all readers and citable by the Digital Object Identifier (DOI®). "Just Accepted" is an optional service offered to authors. Therefore, the "Just Accepted" Web site may not include all articles that will be published in the journal. After a manuscript is technically edited and formatted, it will be removed from the "Just Accepted" Web site and published as an ASAP article. Note that technical editing may introduce minor changes to the manuscript text and/or graphics which could affect content, and all legal disclaimers and ethical guidelines that apply to the journal pertain. ACS cannot be held responsible for errors or consequences arising from the use of information contained in these "Just Accepted" manuscripts. 
1

2

3

4

5

6

7

8 10 11 12 13 14 15 16 17 18

19

20

21

22

23

24

25

26

27

28

29

30

31

32

33

34

35

36

37

38

39

40

41

42

43

44

45

46

47

48

49

50

51

52

53

54

55

56

57

58

59

60

\section{Activation of proton translocation by respiratory Complex I}

\author{
Nikolai Belevich ${ }^{1}$, Christoph von Ballmoos ${ }^{2}$, Marina Verkhovskaya ${ }^{3^{*}}$ \\ 1,3 Institute of Biotechnology, PO Box 65 (Viikinkaari 1) FIN-00014 University of Helsinki, \\ Finland \\ *Author for correspondence: e-mail: Marina.Verkhovskaya@Helsinki.Fi \\ ${ }^{2}$ Department of Chemistry and Biochemistry, University of Bern, Freiestrasse 3, $\mathrm{CH}-3012$ \\ Bern,
}

Switzerland 


\begin{abstract}
Activation of proton pumping by reconstituted and native membrane-bound Complex I was studied using optical electric potential- and $\mathrm{pH}$-sensitive probes. We find, that reconstituted Complex I has a delay in proton translocation, which is significantly longer than the delay in quinone reductase activity, indicating an initially decoupled state of Complex I. Studies of the amount of NADH required for the activation of pumping indicate the prerequisite of multiple turnovers. Proton pumping by Complex I was also activated by NADPH, excluding significant reduction of Complex I or pre-existing $\Delta \psi$ as activation factors. Co-reconstitution of Complex I and ATPase did not indicate an increased membrane permeability for protons in the uncoupled Complex I state. The delay in Complex I proton pumping activation was also observed in subbacterial vesicles. While it is negligible at room temperature, it strongly increases at a lower temperature. We conclude that Complex I undergoes a conversion from decoupled to coupled state upon the activation. The possible origins and importance of the observed phenomenon are discussed.
\end{abstract}

\title{
Introduction
}

$\mathrm{NADH}$ :ubiquinone oxidoreductase (Complex $\mathrm{I}$ ) is the electron input enzyme in the respiratory chain of mitochondria and many bacterial species, which couples electron transfer from NADH to ubiquinone with transmembrane proton translocation. The electron transfer proceeds along the intraprotein redox chain located in the hydrophilic domain and proton translocation is performed by antiporter-like membrane subunits. No redox centers are found in the membrane fragment 
where the antiporter-like subunits are disposed in a row so that the energy of the redox reaction must be transduced over a distance of $100 \AA$. Such high spatial separation of electron and proton transfers with no obvious coupling site indicates an intricate molecular mechanism of proton pumping. Elucidation of this mechanism could be significantly advanced by monitoring the enzyme operation upon turnover in real time, which prerequisites the enzyme in its active state. However, oxidized mitochondrial Complex I exists in a de-active state (D) which spontaneously converts to an active state $(A)$ upon turnover ${ }^{1-3}$, after which the oxidized enzyme slowly relaxes to the Dstate. The D/A transition is believed to be physiologically relevant since it could serve as a protective mechanism preventing the oxidative damage of tissues (see for a review ${ }^{4}$ ). In contrast to mitochondrial complex I, the D/A transition was not observed in bacterial Complex I from Paracoccus denitrificans ${ }^{5}$, Thermus thermophilus and Escherichia coli ${ }^{6}$ and it was suggested that the D/A transition might be a unique property of the former ${ }^{7,8}$. Subunit composition and molecular structures of Complex I from various organisms demonstrate that bacterial enzymes are "minimal versions" of the mitochondrial ones, i.e. they exert the exact same molecular function with similar rates and stoichiometry ${ }^{7-10}$. Bacterial Complex I is thus a good model for studying the coupling mechanism between $\mathrm{NADH}$ oxidation and proton translocation, although it might not share all properties, such as D/A transition. Upon this transition, mitochondrial Complex I is proposed to undergo conformational changes occurring within three subunits at the junction of the hydrophilic and membrane fragments of the enzyme ${ }^{11,12}$. Although the D/A transition has been proposed to be a unique property of the mitochondrial enzyme ${ }^{7,8}$, dynamics and flexibility of the region at the hydrophilic/membrane domain ${ }^{10}$ introduces some uncertainty in modeling of 
the conformational changes involved and interconversion of these states in bacterial enzymes cannot be excluded and must be studied experimentally.

We have recently shown that $E$. coli Complex I may also exist in two states, resting (R) and active (A), characterized by a delayed maximal $\mathrm{NADH}$ :ubiquinone oxidoreductase activity ${ }^{13}$. In a subsequent study using fluorescent probes, we obtained results indicating that upon the activation from state $\mathrm{R}$ to state $\mathrm{A}$, Complex I undergoes conformational changes, which can be mapped to the junction of the hydrophilic and membrane domains in the region of the assumed acetogenin-binding site $^{14}$. The observed R/A transition was quick $(1-2 \mathrm{~s})^{13}$, regardless whether Complex I was solubilized, reconstituted into liposomes or native membrane-bound and thus faster than reported for the mitochondrial enzyme $\left(\tau_{1 / 2} \sim 10 \mathrm{~s}\right)$. The lifetime of $A$ state was too short to be determined, which is in contrast to an active state of several hours in the mitochondrial enzyme at $0^{\circ} \mathrm{C}^{15}$.

In the previous report, R/A transition with solubilized or reconstituted enzyme was analyzed under conditions that neglected the proton pumping activity of Complex $\mathrm{I}^{13}$. Here, we study the activation profiles of proton pumping activity using reconstituted and native membrane-bound Complex I. We find that the activation properties of the two reactions catalyzed by Complex I, $\mathrm{NADH}$ :ubiquinone oxidoreduction and proton translocation across the membrane, are significantly different.

\section{Materials and Methods}


Bacterial growth and purification of Complex I and ATP synthase. The E. coli MWC215 (Sm ${ }^{\mathrm{R}}$ $n d h:: \mathrm{Cm}^{\mathrm{R}}$ ) strain was grown in $\mathrm{LB}$ medium at $37^{\circ} \mathrm{C}$ in a $25 \mathrm{~L}$ fermenter and harvested at the late exponential growth phase. The membranes for Complex I purification were prepared by passing the cells through an APV Gaulin homogenizer. Then Complex I was purified by two consecutive chromatography steps using DEAE-Trisacryl M (Bio-Sepra) anion exchanger columns and gel filtration on Superdex 200 prep grade (GE Healthcare), respectively, as described ${ }^{16}$. ATP synthase of $E$. coli was expressed and purified as described previously ${ }^{17}$

Reconstitution. Purified Complex I was reconstituted into liposomes as previously described ${ }^{18}$ except that azolectin was suspended in the buffer containing MOPS-BTP $100 \mathrm{mM}, \mathrm{pH}$ 7.0, n-dodecyl $\beta$-D-maltopyranoside (DDM) $0.4 \%$, Na-cholate $0.1 \%$. A ratio of $1: 10(w / w)$, of the enzyme to azolectin was used unless other specified. ATP synthase and ATP synthase/Complex I coreconstitution were performed in the same way.

Preparation of subbacterial vesicles. The vesicles were prepared as described in $^{19}$, but vesicles were loaded with $100 \mathrm{mM}$ MOPS-BTP, 7.2, $50 \mathrm{mM} \mathrm{KCl}$ and $1 \mathrm{mM} \mathrm{MgSO}_{4}$, frozen and stored at $80^{\circ} \mathrm{C}$ until use.

\section{Kinetic measurements.}

Monitoring $\Delta \psi$ in proteoliposomes was carried out at $24^{\circ} \mathrm{C}$ using a high-resolution CCD array spectrometer (HR2000+, Ocean Optics) combined with a DH-2000-BAL light source (Ocean Optics). Upon the reaction, optical spectra in the range of $250-680 \mathrm{~nm}$ were collected for $600 \mathrm{~s}$. The difference in absorption at $630-590 \mathrm{~nm}$ presents $\Delta \psi$-sensitive Oxonol VI response. Optical changes at $340 \mathrm{~nm}$ reflect simultaneous $\mathrm{NADH}$ consumption. The assay buffer comprised $100 \mathrm{mM}$ 
MOPS-BTP, 7.0, $1 \mathrm{mM} \mathrm{MgSO}_{4}, 5 \mathrm{mM}\left(\mathrm{NH}_{4}\right)_{2} \mathrm{SO}_{4}, 2.5 \mu \mathrm{M}$ Oxonol VI, $10 \mathrm{nM}$ solubilized bo ${ }_{3}$ oxidase, decylubiquinone (DQ) $100 \mu \mathrm{M}$. Prior to measurements proteoliposomes (protein concentration 7$15 \mu \mathrm{g} / \mathrm{ml}$ ) were incubated in the assay buffer for $7 \mathrm{~min}$ at RT for equilibration of the DQ distribution.

Monitoring $\Delta \mathrm{pH}$ in proteoliposomes were performed using entrapped $\mathrm{pH}$-sensitive probe trisodium 8-hydroxypyrene-1,3,6-trisulfonate (pyranine) as described $\mathrm{in}^{18}$.

Monitoring $\Delta \mathrm{pH}$ in membrane vesicles was carried out following fluorescence changes of $\mathrm{pH}$ sensitive probe acridine orange (AO) with a Hitachi F-7000 fluorescence spectrophotometer, $\lambda_{\mathrm{ex}}=$ $493 \mathrm{~nm}, \lambda_{\mathrm{em}}=530 \mathrm{~nm}$ at $24^{\circ} \mathrm{C}$ unless other specified. The assay buffer comprised $50 \mathrm{mM}$ MOPSBTP, pH 7.2, $100 \mathrm{mM} \mathrm{KCl}, 1 \mathrm{mM} \mathrm{MgCl} 2,1 \mu \mathrm{M}$ valinomycin, $100 \mu \mathrm{M} \mathrm{DQ}, 1 \mathrm{mM}$ KCN, $3.5 \mu \mathrm{M}$ AO. The final protein concentration was $150-180 \mu \mathrm{g} / \mathrm{ml}$.

\section{Fast measurements of reconstituted Complex I NADH:ubiquinone oxidoreductase activity.} Following the initial stage of $\mathrm{NADH}$ oxidation was performed by rapid mixing of equal volumes of solubilized Complex I and buffer containing NADH (Unisoku Stopped-flow RSP-2000 apparatus) using a high-resolution CCD-array spectrometer (HR2000+, Ocean Optics) combined with a Xenon lamp as described $\mathrm{in}^{13}$.

\section{Results}

Delay of pumping activity in resting Complex I. In our previous work, activation of Complex I was followed by monitoring NADH oxidation and quinone reduction ${ }^{13}$. In functional Complex I this reaction is coupled to electrogenic proton transfer across the membrane that results in generation of a $\Delta \psi$ and a $\Delta \mathrm{pH}$. In order to monitor transmembrane events, purified Complex I was 
reconstituted into liposomes and energized by addition of NADH in the presence of DQ, and the generation of $\Delta \psi$ or $\Delta \mathrm{pH}$ was monitored (Figure 1). Accumulation of reduced ubiquinone was prevented by addition of a small amount of quinol oxidase $\mathrm{bo}_{3}$ in detergent micelles, regenerating oxidized ubiquinone without

A

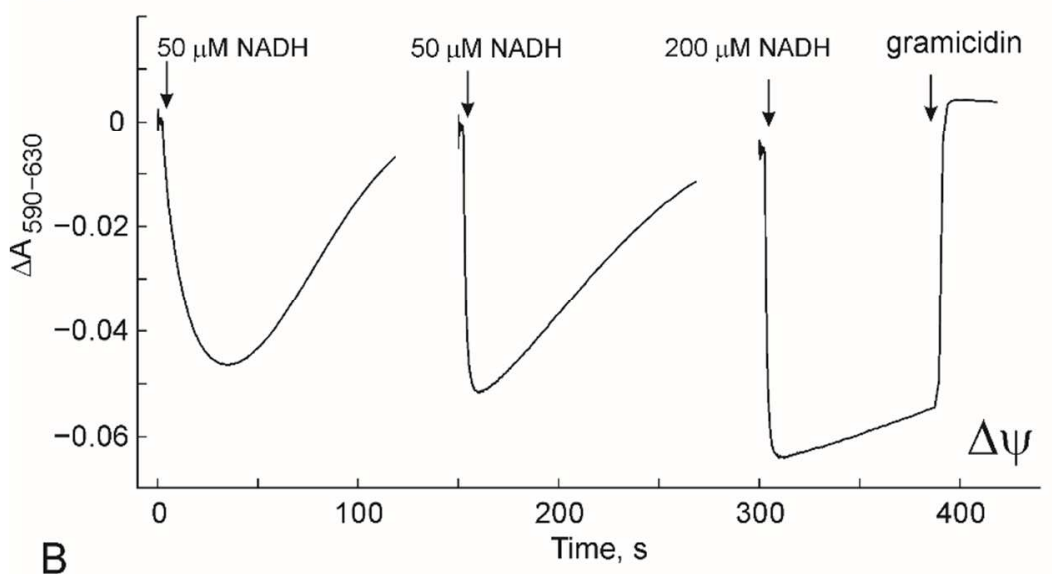

B

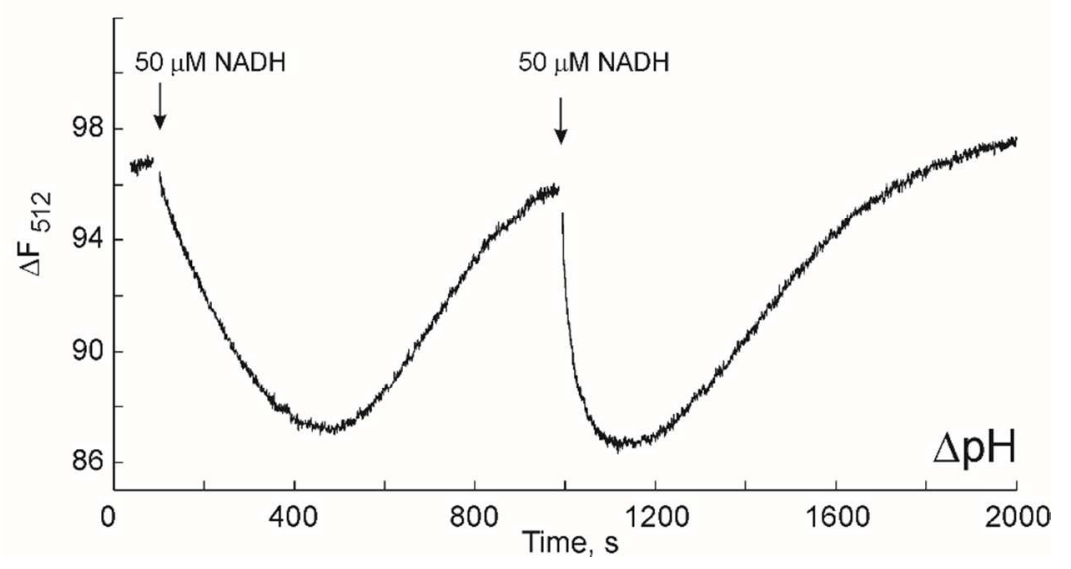

contributing to transmembrane proton transport.
Figure1. Following the $\Delta \psi(\mathrm{A})$ and $\Delta \mathrm{pH} \quad$ (B) generation in proteoliposomes by reconstituted Complex I. Following the first additions of $\mathrm{NADH}$ were made after full dissipation of $\Delta \psi$ or $\Delta \mathrm{pH}$ and complete NADH consumption. $100 \mu \mathrm{M} \mathrm{DQ}$ and $b_{3}$ oxidase was added to proteoliposomes prior to measurements.

Proton pumping initiated by addition of external NADH generates an electric potential (inside positive) across the proteoliposomes membrane that was followed using the $\Delta \psi$-sensitive probe, 
Oxonol VI. As shown in Figure 1A, initial addition of NADH resulted in $\Delta \psi$ generation followed by its relaxation to zero level after all NADH was consumed. Notably, subsequent NADH additions caused much faster formation of $\Delta \psi$. A similar effect was observed when acidification of the liposome lumen was followed with the $\mathrm{pH}$-sensitive dye pyranine entrapped in the liposomes (Figure 1B). Similar to $\Delta \psi$ generation, the second addition of NADH caused a faster rate of acidification than the first addition. While the kinetics of the two experiments are different (fewer protons are required to establish a membrane potential than to significantly acidify the buffered liposome interior), the similar trend strongly indicates that initial proton pumping (in the resting enzyme) is much slower than in the activated enzyme.

Correlation of pumping and NADH:ubiquinone oxidoreductase activity. In the next series of experiments, activation profiles of both NADH oxidation (as reported $\mathrm{in}^{13}$ ) and $\Delta \psi$ generation were compared simultaneously (Figure 2A) by two consecutive NADH additions as described above.
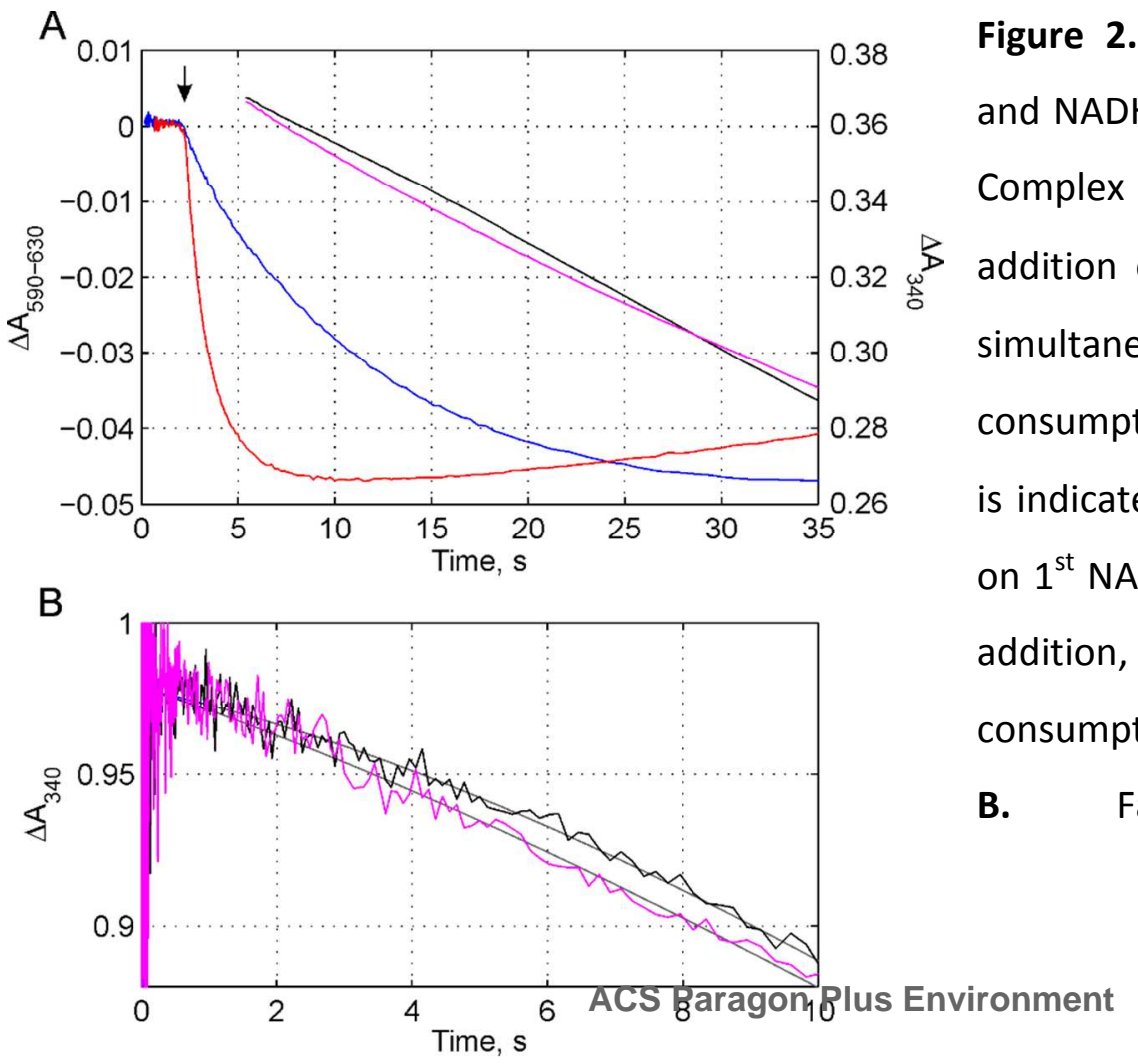

Figure 2. A. Kinetics of $\Delta \psi$ generation and NADH consumption by reconstituted Complex I upon the first and the second addition of $50 \mu \mathrm{M}$ NADH. Upper panel, simultaneous $\Delta \psi \quad$ and NADH consumption monitoring. NADH addition is indicated by an arrow. $\Delta \psi$ generation on $1^{\text {st }} \mathrm{NADH}$ addition, blue, on $2^{\text {nd }} \mathrm{NADH}$ 52 53 54 55 56 57 
NADH:ubiquinone oxidoreduction by reconstituted Complex I. At zero time, the solution containing proteoliposomes equilibrated with $100 \mu \mathrm{M}$ DQ was mixed 1:1 with the same buffer containing $100 \mu \mathrm{M}$ NADH. Non-activated proteoliposomes, black; pre-activated with $30 \mu \mathrm{M}$ NADH, magenta. Gray lines, exponential fits of the kinetics.

The data in Figure $2 \mathrm{~A}$ show that the difference in rates between the first and second addition is much more pronounced for proton pumping than for NADH oxidation. While the NADH consumption rate in the time interval of 5-10 s at the first NADH addition was $\sim 70 \%$ of the second addition, the maximal rate of $\Delta \psi$ response on the first NADH addition was only $\sim 15 \%$ of the second.

The discrepancy between the rates of proton and electron transport indicates that Complex I is partially uncoupled during the first seconds of the measurements, i.e. electron transport is not tightly coupled to transmembrane proton transport. In the used setup, NADH oxidation could not be reliably followed in the time interval of $0-5 \mathrm{~s}$ because of a strong artificial response at $340 \mathrm{~nm}$ upon NADH dilution. To exclude the possibility that the strong activation of NADH consumption occurs during this time range, we performed stopped-flow measurements to resolve the initial time range (Figure 2B). At zero time, suspensions containing proteoliposomes, either untreated or activated with $30 \mu \mathrm{M}$ NADH for several minutes, were mixed 1:1 with the same buffer containing $100 \mu \mathrm{M} \mathrm{NADH}$. The results in Figure $2 \mathrm{~B}$ show that the difference in the initial kinetics of NADH:ubiquinone oxidoreductase activities did not exceed $10 \%$.

Dependence the proton pumping activation on the ratio Complex I:azolectin upon reconstitution. The energization of proteoliposomes membrane is always a result of a superposition of two 
oppositely directed fluxes, one being active $\mathrm{H}^{+}$pumping by the enzyme and the other being $\mathrm{H}^{+}$(or another ion) leakage through the membrane. The signal amplitude of active pumping is thus expected to increase with the number of Complex I proteins in the liposomes, while the membrane leakage contribution remains unaffected (Figure 3A).

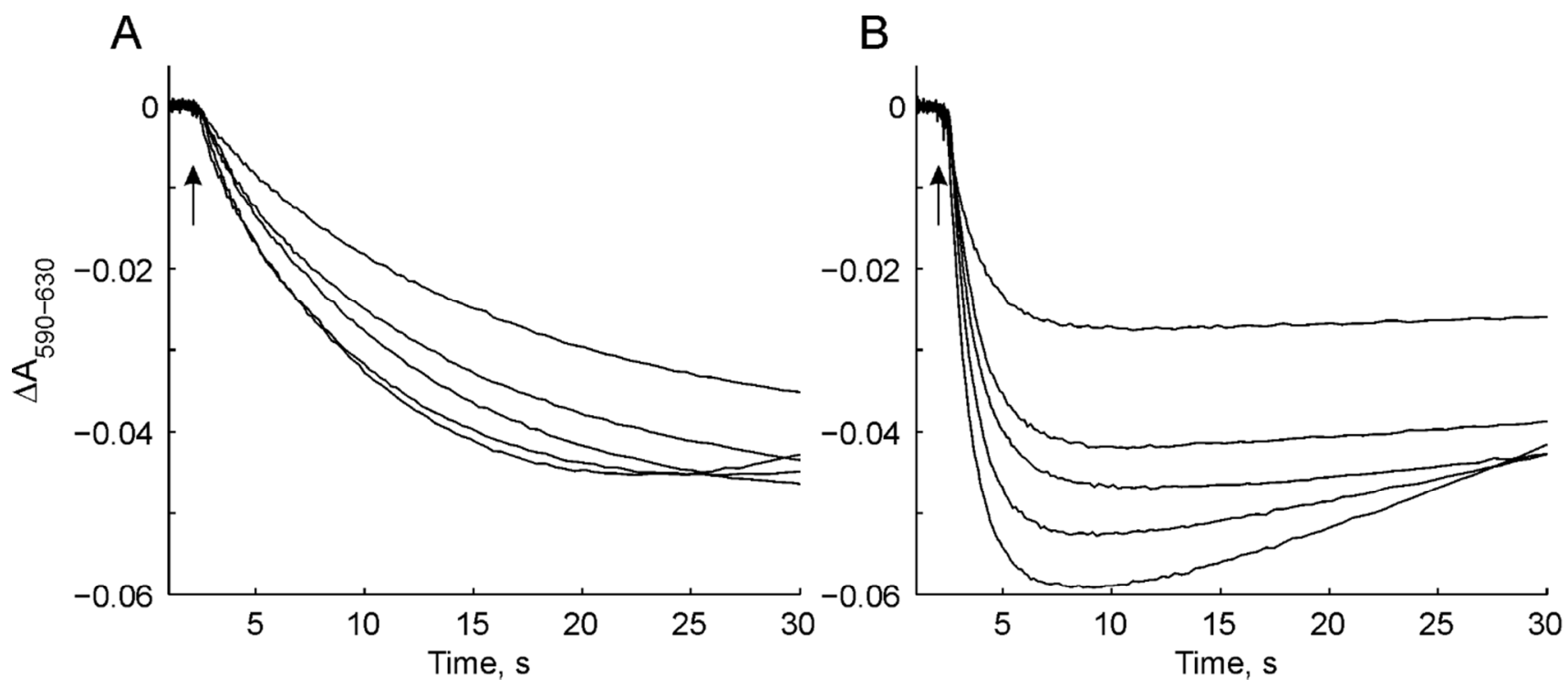

Figure 3. Kinetics of $\Delta \psi$ generation by reconstituted Complex I upon the first $(\mathbf{A})$ and the second (B) addition of $\mathrm{NADH}$ at $50 \mu \mathrm{M}$ concentration indicated by arrows. The ratio Complex I/azolectin (w/w) upon reconstitution 1:50,1:30,1:20,1:15, 1:10, from top to bottom. NADH additions are indicated by arrows.

Purified Complex I preparation contains some amount of annular lipids that could be important for the enzyme behavior. Reconstitution into azolectin liposomes may result in a replacement of these lipids dependently on the proportion of the lipid phase, therefore it was important to test whether proteoliposome content affects the observed activation of Complex I. We carried out monitoring 
$\Delta \psi$ generation in proteoliposomes with different Complex I:lipid ratios. In Figure 3 the responses on $1^{\text {st }}(A)$ and $2^{\text {nd }}(B) \mathrm{NADH}$ addition to proteoliposomes were Complex I was reconstituted in ratios of $1: 50,1: 30,1: 20,1: 15,1: 10(\mathrm{w} / \mathrm{w})$ are presented. Although the amplitude of the response changed in accordance with protein content, as expected, the proportion between the initial rates after $1^{\text {st }}$ and $2^{\text {nd }} \mathrm{NADH}$ additions stayed unaltered. When the ratio Complex I:lipid exceeds 1:10 $(w / w)$ the signal becomes lower, most probably due to Complex I aggregation and poor reconstitution. Taken together, the results show that the activation phenomenon is not affected by changes in protein:lipid ratio.

Dependence of Complex I activation on concentration of initially added NADH. Next, the effect of NADH concentrations varying from 2 to $100 \mu \mathrm{M}$ as the first addition was tested (Figure 4A), while the second addition was always $50 \mu \mathrm{M}$. The second portion was added only after the $\Delta \psi$

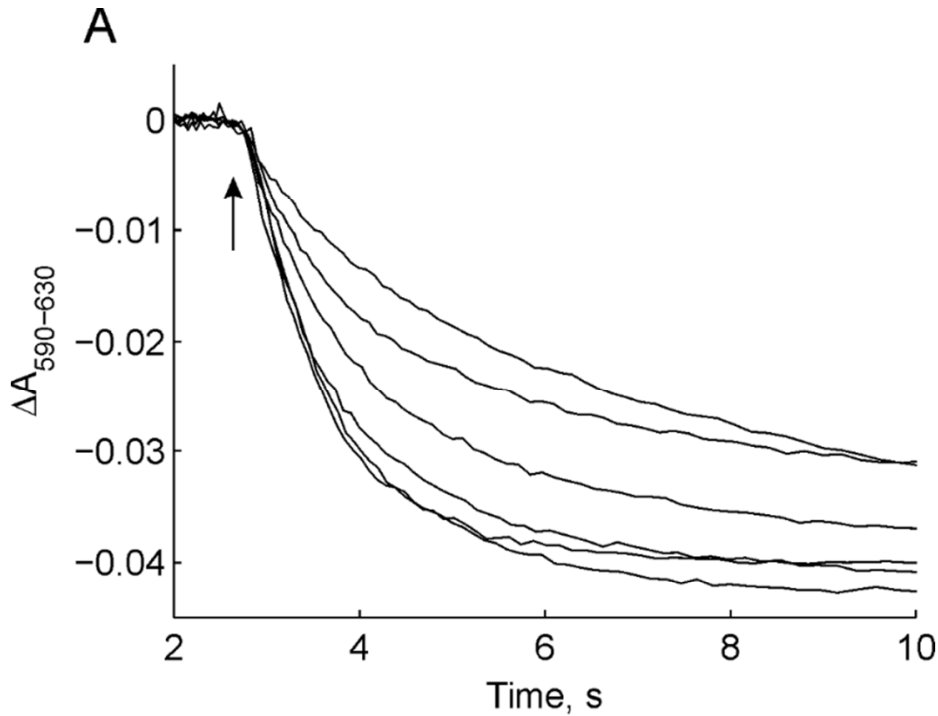

B

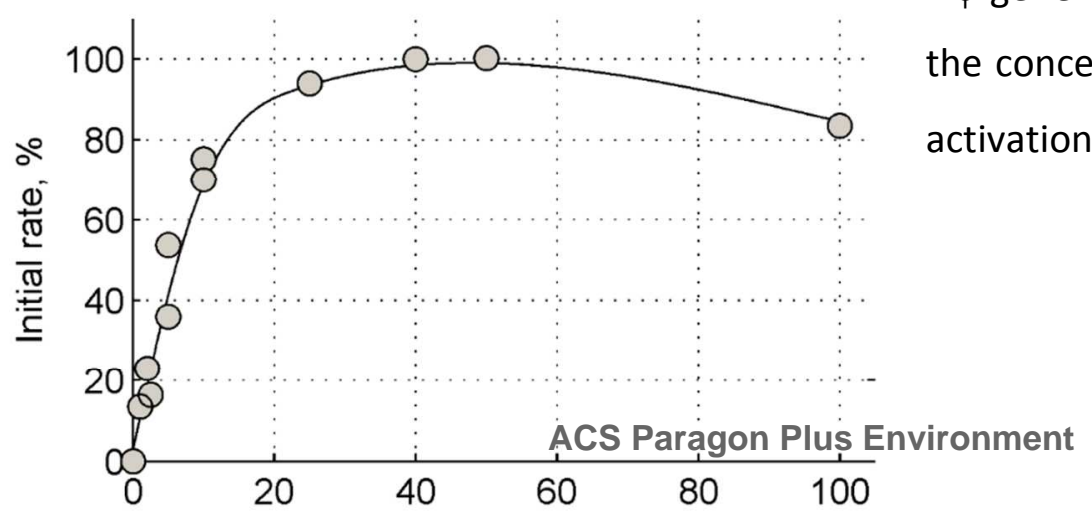

generated

Figure 4. Dependence of reconstituted Complex I activation on concentration of initially added NADH. A. Generation of $\Delta \psi$ on the $2^{\text {nd }} \mathrm{NADH}$ addition at concentration of $50 \mu \mathrm{M}$ (indicated by an arrow). The first NADH addition was 0, 5, $10,20,50,100 \mu \mathrm{M}$, from top to bottom. B. Dependence of the initial rate of $\Delta \psi$ generation by activated Complex I on 51 53 54 55 56 
upon the first addition had completely dissipated. We found that the full activation of proton pumping required 10-15 $\mu \mathrm{M}$ NADH during the first addition, while further increase in NADH concentration resulted in the same activation (Figure 4B). Upon the third NADH addition, which is performed immediately after full NADH consumption of the second addition the profile of $\Delta \psi$ generation was similar to the second NADH addition.

Activation of Complex I pumping activity by NADPH. The results from the experiments above indicate that preceding enzyme turnover directly influences the extent of activation. We therefore tested, if activation is possible with NADPH, which is able to reduce Complex I, showing very slow turnover. NADPH was found to activate mitochondrial Complex $1^{15,20}$. As expected, Complex I incubation with $100 \mu \mathrm{M}$ NADPH resulted in negligible NADPH:ubiquinone oxidoreductase activity and almost non-detectable $\Delta \psi$ generation, since the turnover is so slow that $\mathrm{H}^{+}$pumping cannot overcome the membrane leakage (Figure 5, black

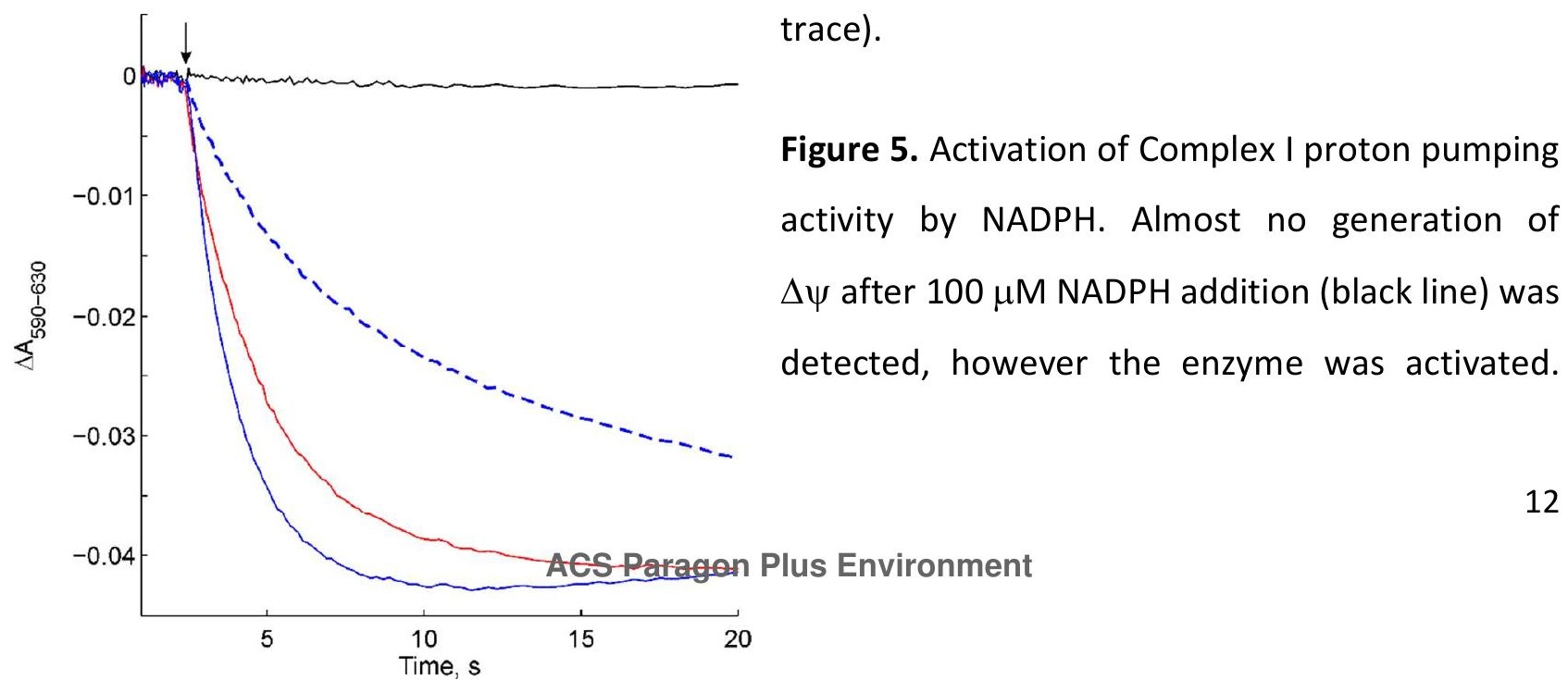


Response to the primary addition of $50 \mu \mathrm{M} N A D H$ after preincubation with NADPH, red. Generation of $\Delta \psi$ without preincubation with NADPH, blue curves: $1^{\text {st }} 50 \mu \mathrm{M}$ NADH addition, dashed; $2^{\text {nd }} 50 \mu \mathrm{M}$ NADH addition, solid. Nucleotides addition is indicated by an arrow.

However, after an incubation of the proteoliposomes with NADPH for 2 minutes, the addition of $50 \mu \mathrm{M}$ NADH shows that Complex I has been significantly (but not completely) activated (Figure 5, red trace) if compared with non-activated complex I (blue dotted trace) or fully activated enzyme (blue trace). Incubation of the proteoliposomes with $100 \mu \mathrm{M} \mathrm{NAD}{ }^{+}$as a substrate analogue that cannot be turned over, did not affect the slow proton pumping on the $1^{\text {st }} \mathrm{NADH}$ addition (Figure S1) and thus not activate Complex I. These data show that the enzyme activation is rather dependent on the turnover than on the incubation with a substrate analogue.

Co-reconstitution of ATPase and Complex I. As mentioned above, a possible explanation for the observed results is that initial NADH oxidation is uncoupled from proton pumping. However, another possibility would be that in the inactive state, Complex I confers a higher proton permeability to the membrane. The observed effect would then be due to a delayed recovery of the low permeability (tightening of the membrane) upon enzyme activation. We tested this hypothesis by co-reconstitution of complex I with ATP synthase, a separate proton pump. In these experiments, ATP is added and the ATP synthase works in hydrolysis direction as a primary proton pump.

As seen in Figure 6 the amplitude and the kinetics of $\Delta \psi$ generated by ATP synthase were similar regardless of whether it was reconstituted alone (blue trace) or together with Complex I, activated (red trace) or non-activated (green trace). The data show that the presence of Complex I in the 
proteoliposomes, either "as prepared" or activated, does not affect the membrane permeability of the liposomes upon ATP driven proton pumping. It should be noted that the amplitude of $\Delta \psi$ generated by the ATP synthase is higher than that of Complex I, which is explained by the lower turnover number of the complex I that is limited by the slow exchange of ubiquinone between lipid and water phase ${ }^{21}$.

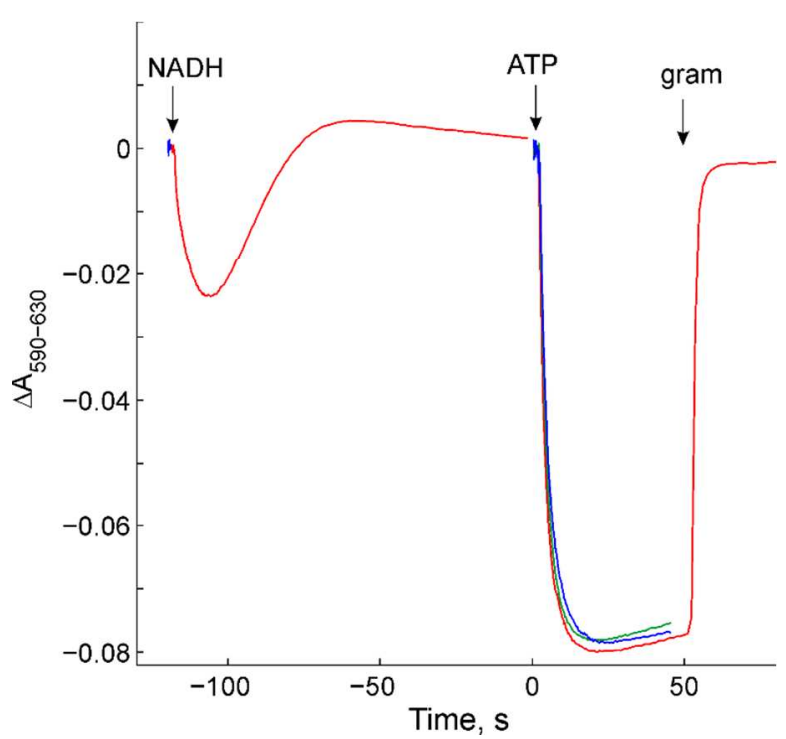

Figure 6. Generation of $\Delta \psi$ by reconstituted ATPase in the presence or absence of Complex I. ATPase reconstituted alone, red curve; ATPase coreconstituted with Complex I, green curve; ATPase was reconstituted with Complex I, which was activated by $50 \mu \mathrm{M} N A D H$, blue curve. $0.4 \mathrm{mM}$ ATP was added as indicated. The dissipation of $\Delta \psi$ was achieved by an addition of $1.5 \mu \mathrm{g} / \mathrm{ml}$ gramicidin $\mathrm{D}$.

Taken together, these data indicate that the different activation profiles of NADH oxidation and proton pumping are indeed due to a lowered proton pumping stoichiometry after the first addition.

Activation of proton pumping by Complex I in membrane vesicles. Finally, inverted membranes vesicles of $E$. coli were prepared to test whether the different activation profiles can also be observed in native membranes. To suppress proton pumping by the $b o_{3}$ oxidase initiated by $\mathrm{NADH}$, the medium was supplemented with $1 \mathrm{mM} \mathrm{KCN}$, which specifically blocks $b_{3}$ oxidase. Instead, $100 \mu \mathrm{M}$ DQ was added as an electron acceptor. In these experiments, only a limited amount of NADH could be used, since the reduced ubiquinone, which is accumulated in the 
absence of $\mathrm{bo}_{3}$ oxidase activity, notably inhibits Complex I, similar to the inhibiting effect of ubiquinol $Q_{1}$ with the mitochondrial Complex $\left.\right|^{22}$. Due to high density and activity of Complex I in the membranes, it was impossible to follow the fast development of $\Delta \psi$, thus we studied proton translocation by following the acidification of the vesicle interior by the $\Delta \mathrm{pH}$ sensitive fluorescent probe acridine orange (AO). To convert $\Delta \psi$ to $\Delta \mathrm{pH}$, the assay buffer was supplemented with $1 \mu \mathrm{M}$

A

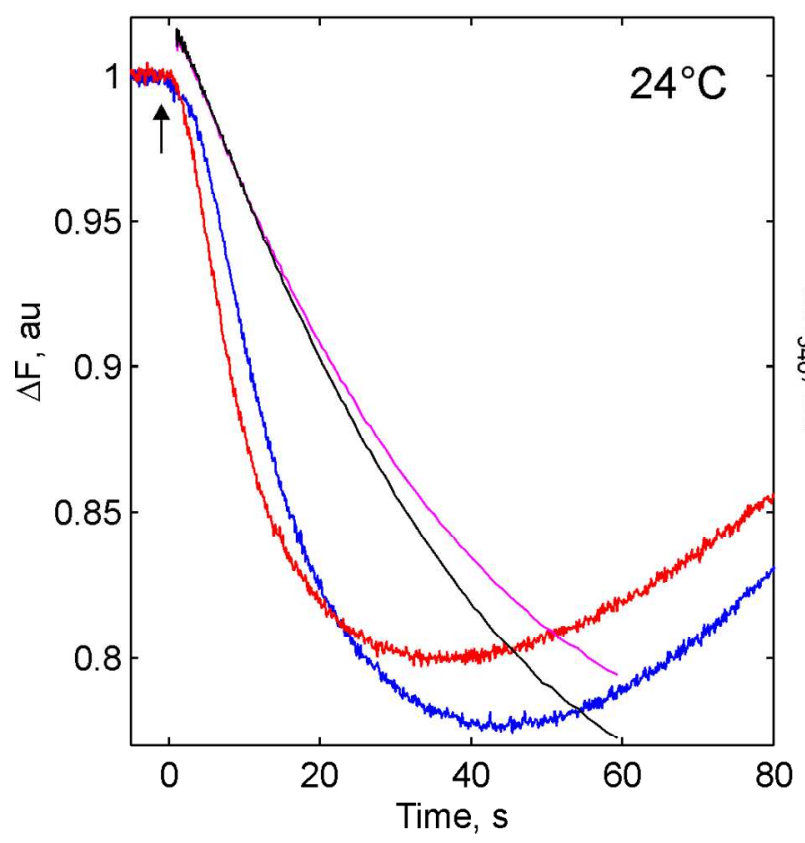

B

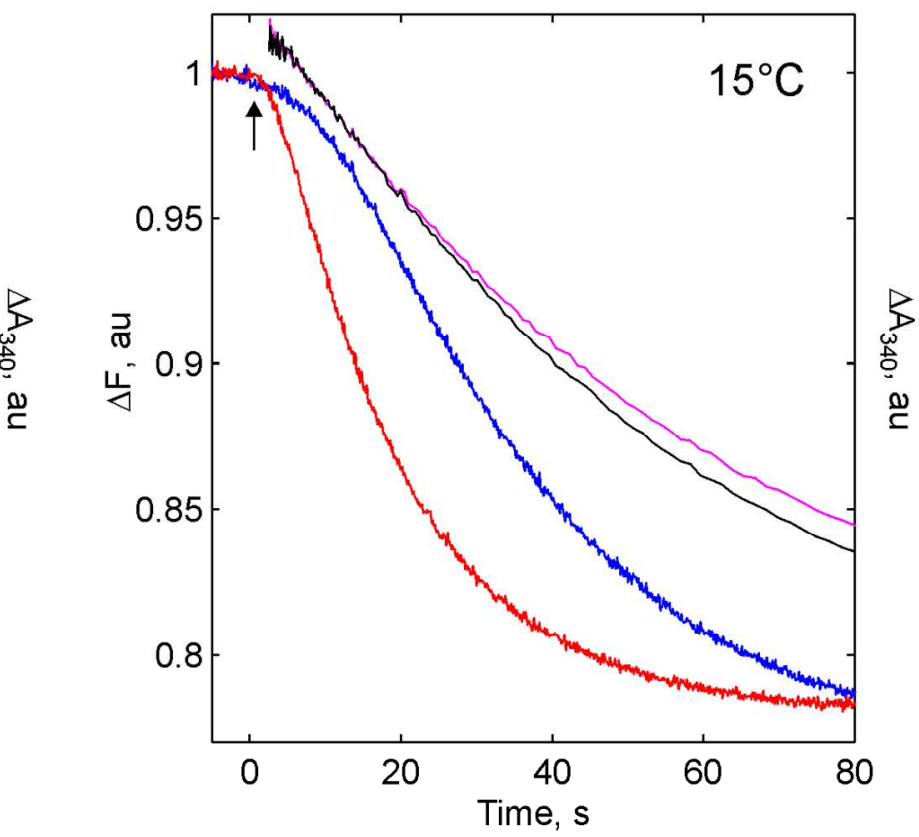

valinomycin and $100 \mathrm{mM} \mathrm{KCl}$. In parallel, NADH consumption was determined optically in separate samples but under identical conditions. At $24{ }^{\circ} \mathrm{C}$, an insignificant acceleration of $\Delta \mathrm{pH}$ generation by pre-activated Complex I was observed and no reliable difference in NADH consumption by nonactivated and activated Complex I was detected as reported previously ${ }^{13}$. However, at lower temperatures the difference in the activity of activated and non-activated Complex I became evident. At $15{ }^{\circ} \mathrm{C}$, the maximal acidification rate is $2-3$ times higher if the enzyme was activated and the delay in pumping is much more pronounced (Figure 7). 
Figure 7. Acidification of the membrane vesicles interior and $\mathrm{NADH}$ consumption at $24{ }^{\circ} \mathrm{C}(\mathbf{A})$ and $15^{\circ} \mathrm{C}$ (B). Before measurements the membranes were equilibrated with $\mathrm{AO}, 100 \mu \mathrm{M}$ DQ and 1 $\mathrm{mM} \mathrm{KCN}$ for $5 \mathrm{~min}$ at corresponding temperatures. AO response to: the $1^{\text {st }} 20 \mu \mathrm{M}$ NADH addition, blue; to the $2^{\text {nd }} 20 \mu \mathrm{M}$ NADH addition, red. NADH consumption at: $1^{\text {st }} 20 \mu \mathrm{M} N A D H$ addition, black; $2^{\text {nd }} 20 \mu \mathrm{M}$ NADH addition, magenta. NADH was added at zero time as indicated by arrow.

NADH consumption as well proceeds with slightly longer delay at lower temperatures, however, it does not correlate with the delay in acidification. The maximal rate of NADH consumption was identical in both conditions, whereas there was still a significant difference in the maximal acidification rates between untreated and activated complex I (Figure 7).

\section{Discussion}

The previously found R/A transition of Complex I from E. coli is a relatively fast process; it occurs in the time range of $1-2 s^{13}$, after which the rate of $\mathrm{NADH}$ :ubiquinone oxidoreduction has reached its steady-state value. Here, we found that in contrast to the full activation of NADH oxidation, coupled proton translocation in reconstituted Complex I stays significantly depressed much longer (Figure 2). Since Complex I contains solvent-accessible, low-potential FMN and therefore produces ROS upon reduction, it could be assumed that at the initial stage of the reaction, accelerated electron leakage to oxygen occurs. Production of ROS at significant rate could result in a mismatch between NADH oxidation and proton pumping at this stage. However, previous measurements of ROS production by Complex I revealed that the rate of ROS production does not exceed $0.2 \%$ of $\mathrm{NADH}$ oxidation rate regardless whether Complex I was solubilized or 
reconstituted $^{23}$. Such low electron leakage cannot affect proton pumping. More likely explanations of the different activation profiles of electron and proton translocations could be that inactive Complex I either provides higher proton permeability to the membrane, or it undergoes a transition from de-coupled to coupled state (D/C transition) upon turnover. In our experiments, the former possibility was excluded by co-reconstitution of Complex I with ATP synthase, a separate proton pump. Irrespective of the presence or the state of Complex I, proton permeability tested by ATP driven proton pumping was unaffected. Based on our findings, we therefore propose that bacterial Complex I undergoes a transition from decoupled to coupled state upon turnover.

Properties of the system did not let us determine the activation time of proton pumping directly. The rate of $\mathrm{NADH}$ consumption may stay at a constant steady state relatively long time if the concentration of substrates is high and of the enzyme is low, allowing the observation of NADH:ubiquinone oxidoreduction activation. In contrast, the membrane energization occurs relatively fast and the final level of $\Delta \mu_{H_{+}}$is approached before the enzyme activation (Figure 1 ). Therefore, only the comparison of the initial rates of the energization reliably indicates the enzyme state. Some information on activation time can be obtained from data on the dependence of full activation of pumping on the amount of NADH. Complex I concentration in the sample was approximately $15 \mathrm{nM}$ and the addition of $2 \mu \mathrm{M}$ NADH guaranteed over 100 turnovers, yet full activation was not achieved. The obtained data showed that the activation can be reached by multiple turnovers during approximately $80 \mathrm{~s}$ required for the consumption of $10 \mu \mathrm{M}$ NADH (Figure 4). The activation of pumping by NADPH also indicates a necessity of multiple turnovers and rules out a requirement of Complex I reduction to the level that can be approached by NADH 
addition. In the presence of $D Q$, the enzyme reduction by NADPH is insignificant due to the much lower affinity of the electron donor NADPH as compared to the electron acceptor DQ. These findings are in agreement with the data on NADPH effect on A/D transition of mitochondrial Complex I: the enzyme reduction by $\mathrm{NADH}$ or $\mathrm{NADPH}$ in the absence of electron acceptors does not initiate its activation, which can be achieved by NADPH only under conditions when electron transfer from the substrate to quinone acceptor is permitted ${ }^{20}$. Pre-existing $\Delta \psi$ maybe also excluded as an essential activation factor, as incubation with NADPH did not result in $\Delta \psi$ generation due to the slow turnover, yet Complex I was activated.

It is likely that the fast acceleration of $\mathrm{NADH}$ :ubiquinone oxidoreduction ${ }^{13}$ and simultaneous $^{2}$ conformational changes $^{14}$ are the early stages of an establishment of the fully functional state of Complex I. The observed lack of direct correlation between electron and proton translocation implies a decoupled state of Complex I that was not detected previously. It is reasonable to raise the question whether this feature is an intrinsic property of Complex I or it is acquired due to enzyme treatment upon purification and reconstitution. To test this, we studied Complex I functioning in native membranes of subbacterial vesicles. Previously we found that $\mathrm{NADH}$ :ubiquinone oxidoreductase activity of the membrane-bound enzyme has a short (1-2 s) delay as well as purified Complex $\mathrm{I}$, and no activation was detected at $\mathrm{RT}^{13}$. In this study, monitoring of the proton pumping activity showed a small, but highly reproducible acceleration after initial activation by $\mathrm{NADH}$. However, lowering the temperature clearly revealed the phenomenon of pumping activation (Figure 7). These results imply that the $D / C$ transition is indeed a built-in property of Complex I, but further studies are required to manifest this statement. 
The proposed transition could be a precursor of active/de-active transition of mitochondrial Complex I, which evolved in evolution to ensure the enzyme's regulation in eukaryotes. Testing proton pumping by Complex I in the natural environment, in membrane vesicles, indicated that D/C transition is an intrinsic property of the enzyme. However, the significantly prolonged decoupled state of the reconstituted enzyme could be of artificial nature, i.e. acquired during the enzyme isolation and purification and might be caused by a loss of an unidentified factor, which provides fast Complex I activation. Nevertheless, it is clear that both native and purified, reconstituted Complex I from E. coli may exist in resting, non-pumping state, which converts to pumping upon slow turnover. Finding of two states of $E$. coli Complex I should be taken into account when planning future research, it provides a powerful tool to determine properties of coupled and decoupled enzyme and, therefore, opens up opportunities for studying the molecular mechanism of Complex I proton translocation.

\section{Funding}

We acknowledge the financial support of the Biocentrum Helsinki 7919530, the Sigrid Juselius Foundation 4702217, and Magnus Ehrnrooth Foundation 96133, and the Swiss National Science Foundation 153351.

\section{Supporting Information}

Figure $\mathrm{S} 1$ showing that $\mathrm{NAD}^{+}$is incapable to activate proton pumping by Complex I.

\section{References}

(1) Gavrikova, E. V., and Vinogradov, A. D. (1999) Active/de-active state transition of the mitochondrial complex I as revealed by specific sulfhydryl group labeling, FEBS Lett. 455, 36-40. 
(2) Gostimskaya, I. S., Cecchini, G., and Vinogradov, A. D. (2006) Topography and chemical reactivity of the active-inactive transition-sensitive $\mathrm{SH}$-group in the mitochondrial NADH : ubiquinone oxidoreductase (Complex I), Biochim. Biophys. Acta 1757, 1155-1161.

(3) Grivennikova, V. G., Kapustin, A. N., and Vinogradov, A. D. (2001) Catalytic activity of NADH-ubiquinone oxidoreductase (Complex I) in intact mitochondria - Evidence for the slow active/inactive transition, J. Biol. Chem. 276, 9038-9044.

(4) Dröse, S., Stepanova, A., and Galkin, A. (2016) Ischemic A/D transition of mitochondrial complex I and its role in ROS generation, Biochim. Biophys. Acta 1857, 946-957.

(5) Kotlyar, A. B., Albracht, S. P. J., and van Spanning, R. J. M. (1998) Comparison of energization of complex I in membrane particles from Paracoccus denitrificans and bovine heart mitochondria, Biochim. Biophys. Acta 1365, 53-59.

(6) Maklashina, E., Kotlyar, A. B., and Cecchini, G. (2003) Active/de-active transition of respiratory complex I in bacteria, fungi, and animals, Biochim. Biophys. Acta 1606, 95-103.

(7) Zickermann, V., Wirth, C., Nasiri, H., Siegmund, K., Schwalbe, H., Hunte, C., and Brandt, U. (2015) Structural biology. Mechanistic insight from the crystal structure of mitochondrial complex I, Science 347, 44-49.

(8) Wirth, C., Brandt, U., Hunte, C., and Zickermann, V. (2016) Structure and function of mitochondrial complex I, Biochim. Biophys. Acta 1857, 902-914.

(9) Fiedorczuk, K., Letts, J. A., Degliesposti, G., Kaszuba, K., Skehel, M., and Sazanov, L. A. (2016) Atomic structure of the entire mammalian mitochondrial complex I, Nature 538, 406-410.

(10) Zhu, J. P., Vinothkumar, K. R., and Hirst, J. (2016) Structure of mammalian respiratory complex I, Nature 536, 354-359.

(11) Galkin, A., Meyer, B., Wittig, I., Karas, M., Schägger, H., Vinogradov, A., and Brandt, U. (2008) Identification of the mitochondrial ND3 subunit as a structural component involved in the active/deactive enzyme transition of respiratory complex I, J. Biol. Chem. 283, 20907-20913.

(12) Babot, M., Labarbuta, P., Birch, A., Kee, S., Fuszard, M., Botting, C. H., Wittig, I., Heide, H., and Galkin, A. (2014) ND3, ND1 and 39kDa subunits are more exposed in the de-active form of bovine mitochondrial complex I, Biochim. Biophys. Acta 1837, 929-939.

(13) Belevich, N., and Verkhovskaya, M. (2016) Resting state of respiratory Complex I from Escherichia coli, FEBS Lett. 590, 1570-1575.

(14) Belevich N, B. G., Chen Z., Sinha S.C., Verkhovskaya M. (2017) Activation of respiratory Complex I from Escherichia coli studied by fluorescent probes, Heliyon 3, e00224.

(15) Kotlyar, A. B., and Vinogradov, A. D. (1990) Slow active inactive transition of the mitochondrial NADHubiquinone reductase, Biochim. Biophys. Acta 1019, 151-158.

(16) Euro, L., Belevich, G., Wikström, M., and Verkhovskaya, M. (2009) High affinity cation-binding sites in Complex I from Escherichia coli, Biochim. Biophys. Acta 1787, 1024-1028.

(17) Wiedenmann, A., Dimroth, P., and von Ballmoos, C. (2008) $\Delta \psi$ and $\Delta \mathrm{pH}$ are equivalent driving forces for proton transport through isolated F-0 complexes of ATP synthases, Biochim. Biophys. Acta 1777, 1301-1310.

(18) Verkhovskaya, M., Knuuti, J., and Wikström, M. (2011) Role of $\mathrm{Ca}^{2+}$ in structure and function of Complex I from Escherichia coli, Biochim. Biophys. Acta 1807, 36-41.

(19) Belevich, G., Euro, L., Wikström, M., and Verkhovskaya, M. (2007) Role of the conserved arginine 274 and histidine 224 and 228 residues in the NuoCD subunit of complex I from Escherichia coli, Biochemistry 46, 526-533.

(20) Vinogradov, A. D., and Grivennikova, V. G. (2001) The mitochondrial complex I: Progress in understanding of catalytic properties, IUBMB Life 52, 129-134. 
(21) Shinkarev, V. P., and Wraight, C. A. (1997) The interaction of quinone and detergent with reaction centers of purple bacteria .1. Slow quinone exchange between reaction center micelles and pure detergent micelles, Biophys. J. 72, 2304-2319.

(22) Grivennikova, V. G., Kapustin, A. N., and Vinogradov, A. D. (2001) Catalytic activity of NADH-ubiquinone oxidoreductase (Complex I) in intact mitochondria - Evidence for the slow active/inactive transition, J. Biol. Chem. 276, 9038-9044.

(23) Knuuti, J., Belevich, G., Sharma, V., Bloch, D.A., Verkhovskaya, M. (2013) A single amino acid residue controls ROS production in the respiratory Complex I from Escherichia coli, Mol. Microbiol. 90, $1190-1200$ 
Activation of proton translocation by respiratory Complex I

Nikolai Belevich, Christoph von Ballmoos, Marina Verkhovskaya

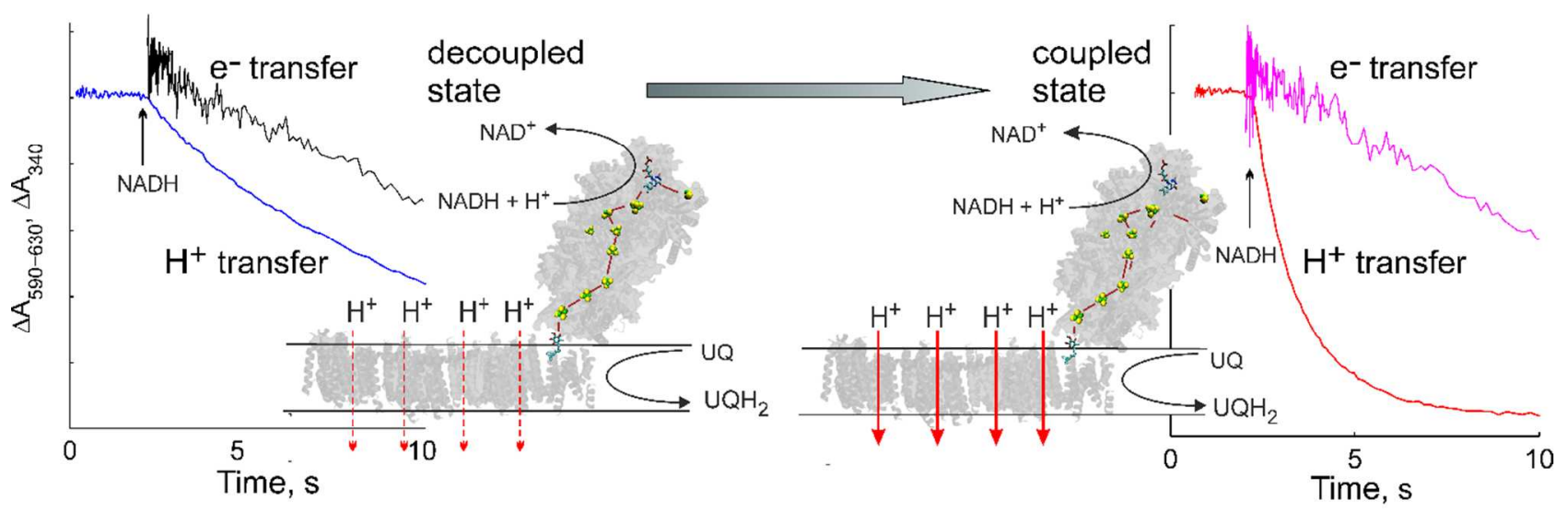

20

21

22

23

24

25

26

27

28

29

30

31

32

33

34

35

36

37

38

39

40

41

42

43

44

45

46

47

48

49

50

51

52

53

54

55

56

57

58

59

60 